\title{
Renal haemodynamics and coronary atherosclerotic burden are associated in patients with hypertension and mild coronary artery disease
}

\author{
GIULIO GERACI ${ }^{1}$, DARIO BUCCHERI ${ }^{2}$, LUCA ZANOLI $^{3}$, PASQUALE FATUZZO $^{3}$, KATIA DI NATALE $^{4}$, \\ MARTA M. ZAMMUTO ${ }^{1}$, EMILIO NARDI ${ }^{1}$, CALOGERO GERACI $^{5}$, ETTORE MANCIA $^{1}$, \\ GIULIA ZAMBELLI ${ }^{4}$, DAVIDE PIRAINO ${ }^{4}$, SALVATORE S. SIGNORELLI ${ }^{6}$, ANTONIO \\ GRANATA $^{7}$, SANTINA COTTONE $^{1}$ and GIUSEPPE MULE ${ }^{1}$

\begin{abstract}
${ }^{1}$ Unit of Nephrology and Hypertension-European Society of Hypertension Excellence Centre, Paolo Giaccone University Hospital, I-90127 Palermo; ${ }^{2}$ Unit of Interventional Cardiology, Hospital San Giovanni di Dio, I-92100 Agrigento; ${ }^{3}$ Department of Clinical and Experimental Medicine, University of Catania, I-95100 Catania; ${ }^{4}$ Department of Interventional Cardiology, Paolo Giaccone University Hospital, I-90100 Palermo; ${ }^{5}$ Unit of Cardiology, S. Elia Hospital, I-93100 Caltanissetta; ${ }^{6}$ Department of Clinical and Experimental Medicine, University of Catania, I-95100 Catania; ${ }^{7}$ Department of Nephrology and Dialysis, Hospital San Giovanni di Dio, I-92100 Agrigento, Italy
\end{abstract}

Received September 1, 2018; Accepted February 11, 2019

DOI: $10.3892 /$ etm.2019.7279

\begin{abstract}
Intrarenal hemodynamic alterations are independent predictors of cardiovascular events in different populations. It has been hypothesized that there is an association between renal hemodynamics and coronary atherosclerotic burden in patients with hypertension. Therefore, the present study examined the associations between renal hemodynamics, coronary atherosclerotic burden and carotid atherosclerotic disease. A total of 130 patients with hypertension aged between 30-80 years who had been referred for an elective coronary angiography were enrolled in the present study. A duplex ultrasound of the intrarenal vasculature was performed to evaluate the resistive index (RI), pulsatility index (PI) and acceleration time (AT). The carotid intima-media thickness was additionally assessed. A coronary angiography was performed to detect the atherosclerotic burden using the Gensini Score (GS). Based on the GS values, subjects were divided into quintiles (I: $\leq 9$; II: 9-17; III: 17-30; IV: 30-44; and V: GS >44) as well as in subjects with mild (GS $\leq 30)$ or severe coronary disease (GS >30). A weak significant difference in PI was identified among quintiles $(\mathrm{P}=0.041)$, whereas, $\mathrm{RI}$ and $\mathrm{AT}$ did not differ
\end{abstract}

Correspondence to: Dr Giulio Geraci, Unit of Nephrology and Hypertension-European Society of Hypertension Excellence Centre, Paolo Giaccone University Hospital, 141 Via del Vespro, I-90127 Palermo, Italy

E-mail: giulio.geraci@unipa.it

Key words: hypertension, coronary artery disease, atherosclerosis, arterial stiffness, renal pulsatility index, renal resistive index, Gensini Score significantly. PI was associated with GS in the group with low coronary atherosclerotic burden ( $\mathrm{GS} \leq 30$; $\mathrm{P}=0.047$ ), whereas, no association was detected in subjects with GS $>30$. This association remained following adjustment for age and left ventricular ejection fraction $(\mathrm{P}=0.025)$. In conclusion, renal vascular alterations were associated with coronary atherosclerotic burden in patients with hypertension with mild coronary disease.

\section{Introduction}

Cardiovascular diseases represent the primary cause of mortality in Western countries, and numerous risk factors, including the reduced glomerular filtration rate, have been associated with greater incidence in myocardial infarction, stroke and sudden cardiac mortality (1-6). Ischemia is a primary pathogenetic factor leading to these events, which represent the final stages of morphofunctional cardiovascular alterations that develop over a long period of time, mostly asymptomatically (7).

Numerous efforts have been made in recent years to search for markers, globally termed subclinical organ damage, to early identify those asymptomatic patients, who already presented a variable combination of vascular, cardiac or renal alterations, thus being at enhanced risk for cardiovascular diseases.

Recently, intrarenal haemodynamics were identified as a potential marker of systemic vascular damage (8) and a useful tool to better stratify the short and long-term cardiovascular risk of patients in different clinical subsets. In particular, renal resistance index (RI) and renal pulsatility index (PI), non-invasively assessed by Duplex ultrasound, were closely associated with well-known markers of subclinical organ damage, including carotid intima-media thickness (cIMT) (9-11), arterial stiffness $(9,12)$ and left ventricular 
mass $(13,14)$. Furthermore, recent studies detected the direct role of intrarenal haemodynamic impairment as an independent predictor of adverse cardiovascular events and mortality in different populations, particularly in the elderly (15) and in patients with chronic kidney disease (CKD), hypertension or heart failure (16-18).

The association between carotid and coronary atherosclerosis has been previously identified (19-23). In the present study, it was hypothesized that the atherosclerotic process additionally affects the renal district and that renal haemodynamics are correlated with coronary atherosclerosis. Therefore, the association between intrarenal vascular alterations with coronary atherosclerotic burden, as well as with carotid atherosclerotic disease, in patients with hypertension was investigated.

\section{Materials and methods}

Study population. The present study involved 130 Caucasian patients, who attended the Department of Cardiology of the University Hospital of Palermo (Palermo, Italy). All subjects aged between 30 and 80 years with a history of hypertension, who were referred for elective coronary angiography, were consecutively enrolled for 18 months from September 2015.

The exclusion criteria included heart failure (New York Heart Association Class IV), permanent atrial fibrillation, heart rate $>100 \mathrm{bpm}$ or $<50 \mathrm{bpm}$, current or previous acute coronary syndrome, previous coronary artery bypass graft, moderate to severe aortic or mitral valve disease, stenosis of renal arteries as assessed by Doppler ultrasound criteria (24), endocrine or malignant hypertension, severe obesity [body mass index $(\mathrm{BMI}) \geq 40 \mathrm{Kg} / \mathrm{m}^{2}$ ], previous surgery or percutaneous interventions on carotid arteries, renal replacement therapy (patients who had a transplant or dialysis), and principal non-cardiovascular diseases. This information had been gathered from medical records and outpatient medical examinations. Written informed consent was obtained from each subject, and the local review board of the University of Palermo approved the study protocol, which conformed to the ethical guidelines of the Helsinki declaration. The datasets used and/or analysed during the current study are available from the corresponding author on reasonable request.

Study design. In all subjects, a comprehensive evaluation of clinical history and physical examination were performed. Patients who reported smoking cigarettes regularly during the past year were considered current smokers. The body weight and height of the patients were measured by a nurse. Clinic blood pressure (BP) was recorded by a doctor, following the recommendations of the 2013 European Society of Hypertension/European Society of Cardiology guidelines (25).

Fasting blood samples were collected to assess routine blood chemistry. A B-mode and Duplex-Doppler ultrasonographic examination of intrarenal vasculature was performed to evaluate RI, PI, and renal acceleration time (AT). Carotid atherosclerotic disease was additionally assessed by Duplex Doppler ultrasound as a model of vascular damage associated with renal parameters, and echocardiography was conducted to prevent bias due to interference of the left ventricular ejection fraction (LVEF), that was calculated using the modified biplane Simpson's rule, according to the American Society of Echocardiography recommendations (26). Later in the same day, a coronary angiography was performed to assess atherosclerotic burden using the Gensini Score (GS) (27).

Measurements. Clinical BP was considered the mean of three consecutive measurements obtained, at 2-min intervals, using an electronic oscillometric validated device (Microlife AG Swiss Corporation, Widnau, Switzerland) (28), after 5 min of rest in the sitting position.

Routine biochemical parameter determination was performed with standard techniques using an autoanalyser (Boehringer Mannheim for Hitachi system 911; Mannheim, Germany). Low-density lipoprotein cholesterol was calculated using the Friedwald formula. Definition and classification of CKD followed the Kidney Disease Improving Global Outcomes guidelines, and the estimated glomerular filtration rate (eGFR) was estimated using the Chronic Kidney Disease-Epidemiology Collaboration equation (29).

Ultrasound evaluation of renal vasculature. The intrarenal Duplex ultrasonography was performed using a GE Logiq P5 PRO instrument (GE Healthcare, Chicago, IL, USA), with a $4 \mathrm{MHz}$ transducer operating at $2.5 \mathrm{MHz}$ for Doppler analysis. The Doppler signal was obtained from the interlobar arteries by placing the sample volume at the level of the cortico-medullary junction $(10,24)$. Peak systolic velocity (PSV), telediastolic velocity (TDV) and mean velocity (MV) were measured. RI was calculated using the formula: $\mathrm{RI}=(\mathrm{PSV}-\mathrm{TDV}) / \mathrm{PSV}$, whereas, PI was obtained using the formula: $\mathrm{PI}=(\mathrm{PSV}-\mathrm{TDV}) / \mathrm{MV}$. The AT was measured as the time distance (msec) between the onset of systolic upstroke with the first systolic peak. The Doppler angle selected was $<60^{\circ}$, and special care was taken not to compress the kidney and to prevent the patient performing a Valsalva maneuver, because these factors may affect measurements. The values were calculated as the average of six measurements from the upper, middle and lower thirds of the two kidneys. Values of RI, PI and AT were $\geq 0.70, \geq 1.20$ and $\geq 100 \mathrm{~ms}$, respectively, and were considered abnormal in agreement with existing literature $(10,24,30,31)$.

Ultrasound evaluation of carotid arteries. The carotid ultrasonographic investigation was performed [using the same GE Logiq P5 PRO instrument (GE Healthcare)] with a 10 $\mathrm{MHz}$ linear-array transducer operating at $5 \mathrm{MHz}$ for Doppler analysis. The examination was obtained following the recommendations of the Mannheim Carotid Intima-Media Thickness Consensus (32).

cIMT was defined as the distance between the vascular lumen-intima interface (internal measurement site) and the media-adventitia transition (external limit). It was obtained on the common carotid artery through the examination of a freezed longitudinal section of $10 \mathrm{~mm}$ at $1 \mathrm{~cm}$ from the bifurcation, at the end-diastolic state, in the proximal and far wall, using lateral, anterior and posterior projections. A total of six measurements was obtained for the right carotid artery and six for the left carotid using the average values (average cIMT). cIMT was not obtained in the presence of a carotid plaque; however, its measurement was shifted proximally to 
the plaque-free site. According to the 2013 European Society of Hypertension/European Society of Cardiology guidelines for the management of arterial hypertension, cIMT $>0.90 \mathrm{~mm}$ was considered abnormal $(25,32)$.

Plaques, whose focal structures encroached into the arterial lumen of $\leq 0.5 \mathrm{~mm}$ or $50 \%$ of the surrounding cIMT value, or demonstrating a cIMT $>1.5 \mathrm{~mm}$, were considered (32). The severity of the carotid stenosis was evaluated according to the European Carotid Surgery Trial criteria (33). A 25\% cut-off was selected to distinguish between subjects with higher or lower carotid atherosclerotic burden $(34,35)$.

Angiographic evaluation of coronary arteries. Selective coronary angiography was obtained by applying Judkins technique in all patients (36). Angiograms were recorded in multiple projections with the biplanar digital cardiac imaging floor-mounted system Artis Zee (Siemens AG, Munich, Germany). Coronary angiograms were assessed by two expert interventional cardiologists (DB and DP) who were blinded to the clinical characteristics of the patients and the Doppler ultrasonographic results. According to the American College of Cardiolgy/American Heart Association guidelines for percutaneous coronary intervention (36), an haemodynamically significant coronary artery stenosis was assumed for a value at least equal to the $50 \%$ of the lumen in any of the principal epicardial vessels, including the left primary coronary artery, left anterior descending artery, left circumflex artery, right coronary artery or their principal branches.

The atherosclerotic burden of coronary arteries was evaluated using the Gensini Score (GS) (27). According to this method, a severity coefficient was assigned for each vessel, according to the degree of stenosis $(0,1,2,4,8,16$ or 32$)$ and the importance of the segment itself ( 5 for the left primary trunk to 0.5 for the most distal segments). Subsequently, total GS was obtained by the sum of the partial results as a global severity index of coronary atherosclerotic disease (27).

Statistical analysis. Statistical analysis was firstly conducted on the entire population $(n=130)$ and on the population divided in quintiles based on GS cut-offs as follows: $\leq 9.0$ (GS I; $n=26$ ); $>9$ and $\leq 17$ (GS II; $n=27$ ); $>17$ and $\leq 30$ (GS III; $\mathrm{n}=25)$; $>30$ and $\leq 44$ (GS IV; $n=26)$; and $>44$ (GS V; n=26). Subsequently, data were analysed in three groups based on the Atherosclerotic-Score (AS) as follows: Absence of plaques (AS I; $n=23$ ); carotid stenosis $<25 \%$ (AS II; $n=64$ ); and carotid stenosis $\geq 25 \%$ (AS III; $n=43$ ).

GS values were further evaluated in the population divided into tertiles of PI (I tertile: PI $<1.30$; II tertile: PI $\geq 1.30$; and $<1.57$; III tertile: $\mathrm{PI} \geq 1.57$ ) and RI (I tertile: $\mathrm{RI}<0.715$; II tertile: $\mathrm{RI} \geq 0.715$ and $<0.755$; and III tertile: $\mathrm{RI} \geq 0.755$ ).

The distribution of variables was analysed by the Kolmogorov-Smirnov test. Continuous variables are presented as the mean \pm standard deviation, and were compared by Students' t-test or analysis of variance with Dunnett's post-hoc test for multiple comparisons, with 'GS I' considered the control group. Categorical variables were presented as numbers (and percentages) and compared with $\chi^{2}$ test and Monte Carlo correction. Variables with non-Gaussian distribution (GS and triglycerides) were expressed as the median and interquartile range because of their skewed distribution, and they were log-transformed to better satisfy distributional assumptions prior to using parametric tests.

The univariate and multivariate associations were tested by simple and multiple linear regression analyses. The strength of the associations among the variables was expressed by the Pearson correlation coefficients (r) and the unstandardised (B) and standardised ( $\beta$ ) multiple regression coefficients. The differences between the Pearson coefficients were assessed by r-to-z Fisher transformation. The association between PI (or alternately RI) and GS was further analysed with the correlation curve using different mathematical models (linear, exponential, logarithmic, quadratic, cubic and sigmoidal). The stepwise multivariate regression analysis was performed considering GS (log-transformed) as an outcome variable, and independent variables, including age, sex, smoking habit, BMI, eGFR, PI (or alternatively RI), serum uric acid, serum glucose levels (or diabetes as a dichotomous variable), high density lipoprotein (HDL)-cholesterol, triglycerides (log-transformed), clinic mean BP, clinic pulse pressure, statin treatment $(0=$ no treatment, $1=$ treatment $)$ and antidiabetic treatment $(0=$ no treatment, $1=$ treatment $)$, were included in the models.

Univariate and multivariate analyses were first conducted on the entire population and subsequently in the two groups; low (GS $\leq 30 ; n=78$ ) or high (GS $>30 ; n=52$ ) coronary atherosclerotic burden. The cut-off of $\mathrm{GS}=30$ was selected according to existing literature (36). These analyses were additionally conducted using similar multivariate models, as appropriate, in the subgroup of subjects with cIMT $\leq 0.90 \mathrm{~mm}(\mathrm{n}=81)$ and in the small group of subjects with no carotid plaques $(n=23)$.

In all multiple regression analyses, a backward stepwise procedure was used, with $\alpha=0.15$ as the cut-off for entry or removal of variables. For all analyses, the null hypothesis was rejected at a two-tailed $\mathrm{P} \leq 0.05$. SPSS software (version 22.0; IBM Corp., Armonk, NY, USA) was used.

\section{Results}

Higher GS quintiles are associated with greater cIMT, a larger percentage of carotid plaques and coronary involvement. Vascular and haemodynamic characteristics are summarised in Table I. Subjects in the higher GS quintiles had a greater cIMT $(\mathrm{P}=0.001)$, a larger percentage of carotid plaques $(\mathrm{P}=0.001)$ and coronary involvement $(\mathrm{P}<0.001)$ compared with those in lower ones. A weak significant difference in PI was identified among quintiles $(\mathrm{P}=0.041)$, whereas, $\mathrm{RI}$ and AT did not differ significantly. Furthermore, the percentages of subjects with $\mathrm{PI} \geq 1.20$ or $\mathrm{RI} \geq 0.70$; however, not with AT $\geq 100 \mathrm{~ms}$, were significantly higher with the higher GS quintiles $(\mathrm{P}=0.033$ and $\mathrm{P}=0.024$, respectively).

Higher tertiles of PI and RI are associated with greater cIMT values. When the entire population was divided into tertiles of PI, greater cIMT values were observed in subjects with higher PI (Fig. 1; Panel A; P=0.001). Similar results were observed by dividing the population into tertiles according to RI values (Fig. 1; Panel B; $\mathrm{P}=0.006$ ). However, no statistically significant difference in GS existed among the groups (Fig. 1; Panel C, D).

Higher values of PI, RI, AT and GS are associated with severe carotid artery disease. Higher values of PI, RI and AT, in 
Table I. Vascular and haemodynamic data of the overall study population and of the population divided into quintiles based on Gensini Score (GS).

\begin{tabular}{|c|c|c|c|c|c|c|c|}
\hline Variables & $\begin{array}{l}\text { Overall study } \\
\text { population } \\
(\mathrm{n}=130)\end{array}$ & $\begin{array}{c}\text { GS I }(\leq 9) \\
(n=26)\end{array}$ & $\begin{array}{c}\text { GS II (9-17) } \\
\quad(n=27)\end{array}$ & $\begin{array}{l}\text { GS III }(17-0) \\
\quad(n=25)\end{array}$ & $\begin{array}{c}\text { GS IV (30-44) } \\
(n=26)\end{array}$ & $\begin{array}{c}\text { GS V }(>44) \\
\quad(n=26)\end{array}$ & P-value \\
\hline Gensini Score & $23(10-38)$ & $6(3-7)$ & $11(10-12)^{\mathrm{b}}$ & $22(19-26)^{\mathrm{c}}$ & $35(31-38)^{\mathrm{c}}$ & $53(46-60)^{\mathrm{c}}$ & $<0.001$ \\
\hline $\mathrm{CAD}^{\$}, \mathrm{n}(\%)$ & $74(57)$ & $0(0)$ & $3(11)^{\mathrm{a}}$ & $19(76)^{\mathrm{c}}$ & $26(100)^{\mathrm{c}}$ & $26(100)^{\mathrm{c}}$ & $<0.001$ \\
\hline LVEF, \% & $52 \pm 11$ & $53 \pm 13$ & $53 \pm 8$ & $55 \pm 8$ & $51 \pm 11$ & $50 \pm 12$ & 0.709 \\
\hline PI & $1.48 \pm 0.31$ & $1.32 \pm 0.26$ & $1.52 \pm 0.35^{\mathrm{a}}$ & $1.57 \pm 0.25^{b}$ & $1.46 \pm 0.36$ & $1.51 \pm 0.27^{\mathrm{a}}$ & 0.041 \\
\hline RI & $0.74 \pm 0.06$ & $0.71 \pm 0.06$ & $0.75 \pm 0.06$ & $0.75 \pm 0.05$ & $0.73 \pm 0.06$ & $0.75 \pm 0.07$ & 0.090 \\
\hline AT (ms) & $117 \pm 48$ & $122 \pm 48$ & $123 \pm 45$ & $107 \pm 46$ & $118 \pm 63$ & $115 \pm 38$ & 0.843 \\
\hline $\mathrm{PI} \geq 1.20, \mathrm{n}(\%)$ & $110(85)$ & $16(62)$ & $23(85)$ & $24(96)^{\mathrm{a}}$ & $22(85)$ & $25(96)^{\mathrm{a}}$ & 0.033 \\
\hline $\mathrm{RI} \geq 0.70, \mathrm{n}(\%)$ & $97(75)$ & $11(42)$ & $24(89)^{\mathrm{a}}$ & $22(88)^{a}$ & $19(73)^{\mathrm{NS}}$ & $22(85)$ & 0.024 \\
\hline $\mathrm{AT} \geq 100 \mathrm{~ms}, \mathrm{n}(\%)$ & $80(62)$ & $18(69)$ & $17(63)$ & $14(56)$ & $14(54)$ & $17(65)$ & 0.894 \\
\hline cIMT, mm & $0.87 \pm 0.17$ & $0.76 \pm 0.13$ & $0.83 \pm 0.16$ & $0.92 \pm 0.15^{\mathrm{c}}$ & $0.91 \pm 0.18^{b}$ & $0.92 \pm 0.18^{c}$ & 0.001 \\
\hline $\mathrm{cIMT}>0.90 \mathrm{~mm}, \mathrm{n}(\%)$ & $49(38)$ & $2(8)$ & $9(33)^{\mathrm{NS}}$ & $14(56)^{\mathrm{c}}$ & $12(46)^{\mathrm{a}}$ & $12(46)^{\mathrm{a}}$ & 0.009 \\
\hline Carotid plaques, n (\%) & $107(82)$ & $18(69)$ & $21(78)^{\mathrm{NS}}$ & $18(72)$ & $24(92)^{\mathrm{a}}$ & $26(100)^{\mathrm{b}}$ & 0.007 \\
\hline
\end{tabular}

CAD, coronary artery disease; GS, gensini score; LVEF, left ventricular ejection fraction; PI, renal pulsatility index; RI, renal resistive index; AT, renal acceleration time; cIMT, carotid intima-media thickness. ${ }^{\$}$ coronary artery with stenosis $\geq 50 \%$. Dunnett post-hoc test (GS I as control group): ${ }^{a} \mathrm{P}<0.05 ;{ }^{b} \mathrm{P} \leq 0.01 ;{ }^{\mathrm{c}} \mathrm{P} \leq 0.001$. Data are reported as median (interquartile range), mean \pm standard deviation or number (\%).


Figure 1. (A and B) Carotid intima-media thickness (mean values and standard error) and (C and D) Gensini Score (median and interquartile range) in the population divided into tertiles of renal pulsatility index, and renal resistance index. ${ }^{*} \mathrm{P}<0.001$ vs. Tertile 1 .

addition to an increased GS, were additionally observed in patients with severe carotid artery disease (AS III) compared with subjects belonging to AS I and AS II (Fig. 2; Panel A-D; $\mathrm{P}<0.001$ for PI; RI and GS; $\mathrm{P}=0.011$ for $\mathrm{AT}$ ). 

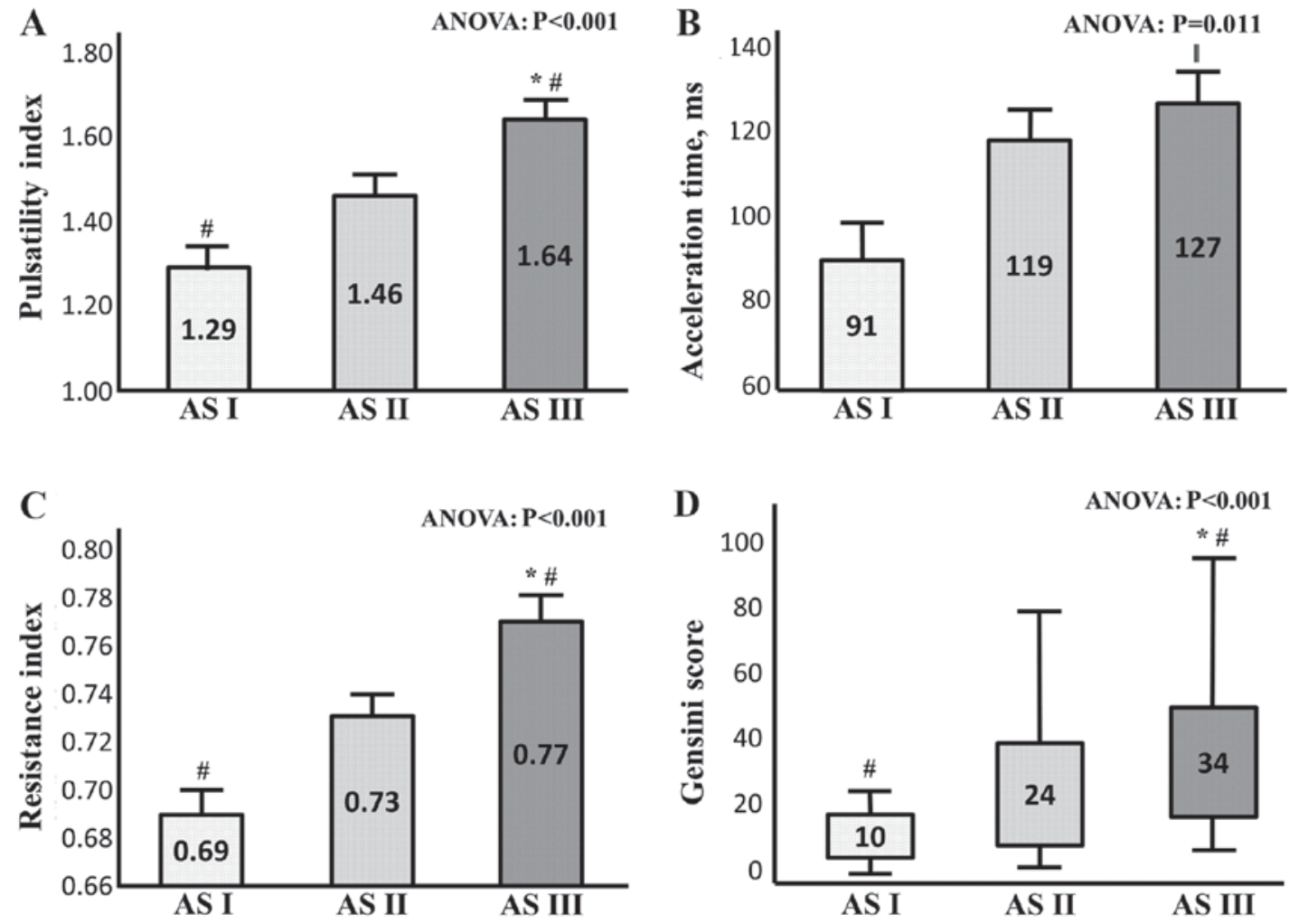

Figure 2. (A) Renal pulsatility index, (B) acceleration time, (C) resistance index and (D) Gensini Score in patients without plaques (AS I), carotid stenosis $<$ $25 \%$ (AS II) and carotid stenosis $\geq 25 \%$ (AS III). AS, Atherosclerotic Score. " $\mathrm{P}<0.001$ vs. Tertile 1 ; ${ }^{\text {II }} \mathrm{P}<0.05$ vs. Tertile 1 ; ${ }^{*} \mathrm{P}<0.05$ vs. Tertile 2.

Low GS is positively correlated with uric acid and triglyceris, and negatively correlated with HDL. Table II demonstrated the correlations between GS with other variables in the whole study population and in the groups, based on severity of coronary (GS $\leq 30$ or GS $>30$ ) or carotid (subjects without or with carotid plaques) atherosclerotic disease; uric acid and triglycerides were positively associated with were correlated with GS in patients with low GS, and HDL was negatively correlated; however, these correlations were not observed in patients with high GS. PI and RI (in addition to AT) did not significantly correlate with GS in entire population by linear univariate analysis, even following adjustment for age and LVEF, and no differences were identified when these associations were alternately evaluated in patients divided for eGFR ( $\geq$ or $<60 \mathrm{ml} / \mathrm{min} / 1.73 \mathrm{~m}^{2}$ ) or diabetes (absence or presence). On the contrary, cIMT was significantly associated with PI ( $\mathrm{r}=0.294 ; \mathrm{P}<0.001), \mathrm{RI}(\mathrm{r}=0.338 ; \mathrm{P}<0.001)$ and GS $(r=0.310 ; \mathrm{P}<0.001)$; however, not with AT.

GS is associated with PI in patients with low coronary atherosclerotic burden. When the association between GS and renal haemodynamic indices was tested using different mathematical models, the best resulting model was the one between GS and PI, as presented in Fig. 3. Due to the shape of the curve, this association in the two groups at low $(\mathrm{GS} \leq 30)$ or high (GS >30) coronary atherosclerotic burden was separately analysed. GS was demonstrated to be statistically associated with PI (however, not to RI or AT) only in the group with GS $\leq 30(\mathrm{r}=0.226 ; \mathrm{P}=0.047)$. This association was more significant in subjects with cIMT $\leq 0.90 \mathrm{~mm}(\mathrm{r}=0.249 ; \mathrm{P}=0.025)$, and it was additionally maintained in the small subgroup of subjects with no carotid plaques $(\mathrm{r}=0.511 ; \mathrm{P}=0.013)$.
PI does not independently correlate with GS; however, is associated with GS. From the multivariate analysis, PI (in addition to RI) did not independently correlate with GS in the overall study (Table III); however, it was independently associated with GS when multivariate analyses were performed alternatively in the group with GS $\leq 30$ (Table III) or in subjects with cIMT $\leq 0.90 \mathrm{~mm}$ (Table III), even if the standardized regression coefficients of PI were not particularly high.

\section{Discussion}

The primary result of the present study is that the intrarenal vascular alterations were significantly correlated with coronary artery disease in patients with low atherosclerotic burden (GS $\leq 30$ or cIMT $\leq 0.90 \mathrm{~mm}$ ), whereas, this association was not identified in those with severe coronary artery disease (GS >30).

A number of previous studies demonstrated that renal haemodynamic alterations may represent the local epiphenomena of early damage of the systemic vascular bed in patients with hypertension; however, renal haemodynamic impairment was able to promote early atherosclerotic damage itself, thus, resulting in a self-perpetuating process $(9,10)$. In 356 asymptomatic patients with hypertension, with or without impairment in renal function, it was previously demonstrated that RI strongly correlated with subclinical carotid atherosclerosis (10), and numerous previous studies demonstrated that intrarenal vascular alterations were associated with other early markers of organ damage (including left ventricular mass, aortic pulse wave velocity or microalbuminuria), in the absence of overt clinical involvement $(9,11,12,14)$. 
Table II. Main correlations in the entire study population, in the groups with GS $<30$ or GS $\geq 30$, and in the 2 groups without or with carotid plaques.

\begin{tabular}{|c|c|c|c|c|c|}
\hline \multirow[b]{2}{*}{ Variables } & \multicolumn{5}{|c|}{ Gensini score (GS) } \\
\hline & $\begin{array}{l}\text { Overall } \\
\text { population } \\
(\mathrm{n}=130)\end{array}$ & $\begin{array}{c}\mathrm{GS} \leq 30 \\
(\mathrm{n}=78)\end{array}$ & $\begin{array}{c}\mathrm{GS}>30 \\
(\mathrm{n}=52)\end{array}$ & $\begin{array}{l}\text { Subjects without } \\
\text { carotid plaques } \\
(\mathrm{n}=23)\end{array}$ & $\begin{array}{c}\text { Subjects with } \\
\text { carotid plaques } \\
(\mathrm{n}=107)\end{array}$ \\
\hline Age & -0.046 & 0.017 & 0.215 & 0.410 & -0.189 \\
\hline Height & 0.154 & 0.176 & -0.152 & 0.114 & 0.187 \\
\hline Weight & 0.074 & 0.032 & 0.019 & 0.201 & 0.187 \\
\hline BMI & 0.004 & -0.094 & 0.171 & 0.235 & 0.097 \\
\hline Glucose & 0.060 & -0.175 & 0.076 & 0.222 & $0.227^{\mathrm{a}}$ \\
\hline Uric acid & 0.022 & $0.276^{\mathrm{a}}$ & 0.004 & 0.403 & 0.054 \\
\hline Total cholesterol & $-0.182^{\mathrm{a}}$ & -0.207 & -0.109 & -0.070 & -0.161 \\
\hline LDL-c & -0.037 & -0.075 & -0.202 & -0.056 & 0.007 \\
\hline HDL-c & $-0.37^{\mathrm{c}}$ & $-0.573^{\mathrm{c}}$ & 0.040 & -0.339 & $-0.330^{\mathrm{c}}$ \\
\hline Tryglicerides & $0.176^{\mathrm{a}}$ & $0.356^{\mathrm{b}}$ & 0.077 & -0.047 & 0.125 \\
\hline Serum creatinine & -0.097 & -0.111 & 0.003 & 0.248 & 0.100 \\
\hline eGFR & 0.079 & 0.067 & -0.156 & -0.130 & -0.023 \\
\hline Hemoglobin & 0.133 & $0.236^{\mathrm{a}}$ & -0.083 & 0.102 & 0.179 \\
\hline Systolic BP & 0.043 & 0.166 & -0.059 & 0.318 & -0.130 \\
\hline Diastolic BP & $0.182^{\mathrm{a}}$ & $0.280^{\mathrm{a}}$ & -0.062 & $0.586^{\mathrm{b}}$ & 0.079 \\
\hline Mean BP & 0.122 & $0.241^{\mathrm{a}}$ & -0.063 & $0.513^{\mathrm{a}}$ & -0.026 \\
\hline Pulse Pressure & -0.080 & 0.019 & -0.043 & -0.147 & $-0.248^{b}$ \\
\hline Heart Rate & -0.048 & -0.120 & 0.098 & 0.254 & -0.085 \\
\hline LVEF & -0.168 & -0.086 & 0.007 & -0.163 & -0.106 \\
\hline PI & 0.136 & $0.226^{\mathrm{a}}$ & 0.108 & $0.511^{\mathrm{a}}$ & 0.084 \\
\hline RI & 0.138 & 0.201 & 0.133 & 0.380 & 0.053 \\
\hline AT & -0.031 & -0.137 & 0.088 & -0.343 & -0.090 \\
\hline cIMT & $0.310^{\mathrm{c}}$ & $0.345^{\mathrm{b}}$ & -0.097 & 0.371 & $0.244^{\mathrm{a}}$ \\
\hline
\end{tabular}

GS, gensini score; BMI, body mass index; LDL-c, low density lipoprotein cholesterol; HDL-c, high density lipoprotein cholesterol; eGFR, estimates glomerular filtration rate; BP, blood pressure; LVEF: left ventricular ejection fraction; PI, (renal) pulsatility index; RI, (renal) resistive index; AT, (renal) acceleration time; cIMT, carotid intima-media thickness. ${ }^{a} \mathrm{P}<0.05 ;{ }^{\mathrm{b}} \mathrm{P} \leq 0.01 ;{ }^{\mathrm{c}} \mathrm{P} \leq 0.001$.

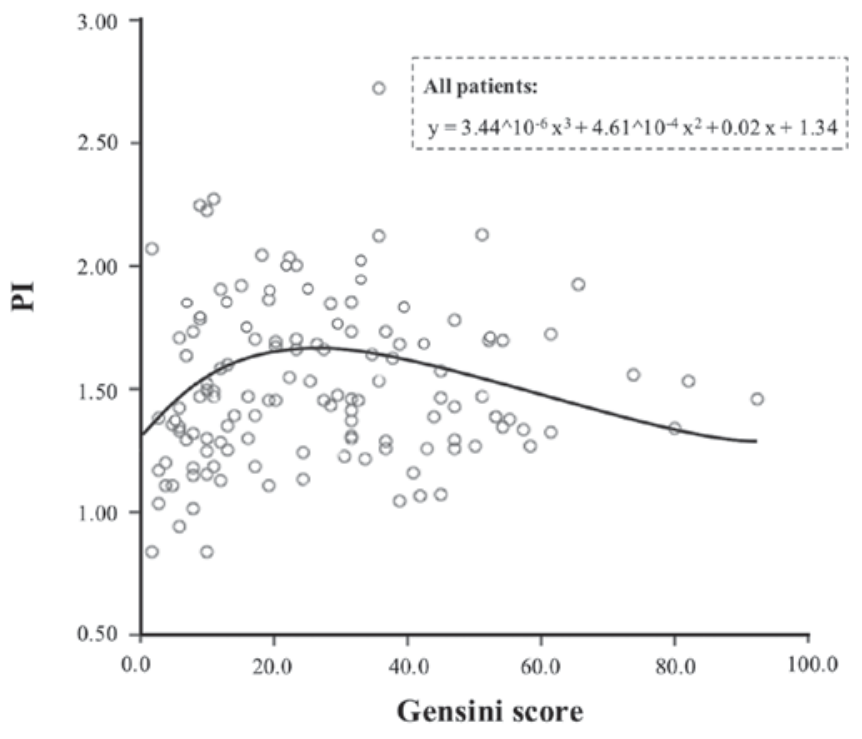

Figure 3. Relationship between Gensini Score and renal pulsatility index (PI) by cubic mathematical model in the overall study population.
Furthermore, renal haemodynamic alterations may reflect systemic vascular damage $(38,39)$, even regardless of hypertension (40), and they may occur at a very early stage in the temporal evolution of atherosclerotic damage. In this regard, Florczak et al (41) observed an independent association between RI and cIMT in healthy subjects, and a limited value of RI in differentiating patients with uncomplicated hypertension from healthy controls. In the present study, PI and RI correlated with cIMT in the overall study population (in agreement with existing literature); however, this association was not observed in the subgroup of subjects with carotid plaques determining stenosis $\geq 25 \%$. Similarly, in the present study, a significant association between PI (or RI) and GS was only identified in the group of patients with lower coronary atherosclerotic burden (and with an earlier vascular damage), and this may additionally explain why the independent association between PI and GS is weak in subjects with early vascular damage, as demonstrated by not particularly high values of standardised regression coefficients of PI at multivariate analyses. 
Table III. Independent multivariate correlates of Gensini Score (log-transformed) in the overall study population (A), in the group of patients with GS $\leq 30(\mathrm{~B})$ and in subjects with cIMT $\leq 0.90 \mathrm{~mm}(\mathrm{C}){ }^{\mathrm{a}}$

A, Model $\left(\mathrm{R}^{2}=0.245\right)$

\begin{tabular}{lccr}
\hline & \multicolumn{3}{c}{ Regression coefficients } \\
\cline { 2 - 3 } & \multicolumn{2}{c}{ Not standardised } & $\frac{3}{\text { Standardised }}$ \\
\cline { 2 - 4 } Outcome variable: Gensini score & $\mathrm{B}$ & $\mathrm{SE}$ & $\beta$ \\
\hline Clinic systolic BP & 0.005 & 0.002 & 0.251 \\
Gender & 0.188 & 0.066 & 0.236 \\
Statins & 0.164 & 0.059 & 0.002 \\
\hline
\end{tabular}

B, Model $\left(\mathrm{R}^{2}=0.454\right)$

\begin{tabular}{|c|c|c|c|c|}
\hline \multirow[b]{3}{*}{ Outcome variable: Gensini score } & \multicolumn{3}{|c|}{ Regression coefficients } & \multirow[b]{3}{*}{$\mathrm{P}$-value } \\
\hline & \multicolumn{2}{|c|}{ Not standardised } & \multirow{2}{*}{$\frac{\text { Standardised }}{\beta}$} & \\
\hline & $\mathrm{B}$ & SE & & \\
\hline HDL-c & -0.009 & 0.002 & -0.425 & $<0.001$ \\
\hline Statins & 0.182 & 0.054 & 0.321 & 0.001 \\
\hline Smoke & -0.222 & 0.072 & -0.304 & 0.003 \\
\hline Serum glucose & -0.002 & 0.001 & -0.273 & 0.007 \\
\hline PI & 0.206 & 0.090 & 0.222 & 0.025 \\
\hline
\end{tabular}

C, Model $\left(\mathrm{R}^{2}=0.205\right)$

\begin{tabular}{|c|c|c|c|c|}
\hline \multirow[b]{3}{*}{ Outcome variable: Gensini score } & \multicolumn{3}{|c|}{ Regression coefficients } & \multirow[b]{3}{*}{ P-value } \\
\hline & \multicolumn{2}{|c|}{ Not standardised } & \multirow{2}{*}{$\frac{\text { Standardised }}{\beta}$} & \\
\hline & B & SE & & \\
\hline Sex & 0.207 & 0.083 & 0.258 & 0.014 \\
\hline PI & 0.274 & 0.124 & 0.226 & 0.030 \\
\hline Statins & 0.173 & 0.081 & 0.225 & 0.035 \\
\hline
\end{tabular}

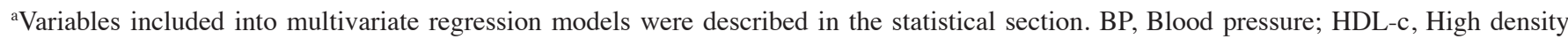
lipoprotein cholesterol; PI, pulsatility index; RI, resistive index; BMI, Body Mass Index; cIMT, carotid Intima-Media Thickness; eGFR, estimated Glomerular Filtration Rate.

In this regard, it is of interest that the enrolled subjects were referred for elective coronary angiography, and thus they represent patients with overt vascular disease and with high or very high cardiovascular risk. Consequently, a number of factors may have been implicated in the pathogenesis of coronary artery plaques. The present results suggested that the renal and coronary haemodynamic impairment proceeds in parallel in the early steps of atherosclerotic process, whereas, other factors have a pivotal role in more advanced coronary artery disease. Alternatively, the greatest variations of the intrarenal haemodynamic indices, even within the normal ranges, may occur prior to the development of a significant atherosclerotic coronary burden and may represent an early step of cardiorenal syndrome type 4 , and chronic abnormalities in renal function may lead to cardiac disease (42). In support of this hypothesis, a $\mathrm{PI} \geq 1.20$ (or a $\mathrm{RI} \geq 0.70$ ) was already present in $61.5 \%$ (or $42.3 \%$ ) of the subjects belonging to the first quintile of GS (GS $\leq 9)$, and the majority of the subjects with GS $\leq 30$ had renal haemodynamic indices above the normal values (PI: 80.8\%; RI: 73.1\%). Furthermore, patients with GS $\leq 30$ had a large percentage of carotid plaques $(73.1 \%)$, whereas, a low atherosclerotic coronary involvement (28.2\%) was present.

The present study has certain potential limitations. Unlike carotid ultrasound, GS does not allow identification of early 
coronary alterations, and the smallest coronary damage that may be detected through GS is a lumen stenosis of 25\% (27). Furthermore, identical structural damage (or identical degrees of stenosis) may result in different scores when located in different areas of the coronary district (27) and this may distort the results and partly explain the lack of correlation between intrarenal haemodynamics and coronary atherosclerosis burden in patients with high GS. However, when the population was divided into groups only based on the number of coronary arteries involved, regardless of the degree and location of the stenosis, results were confirmed. The cross-sectional design of the present study does not allow complete clarification of the association between the intrarenal vascular alterations and coronary atherosclerotic damage. Therefore, an ongoing longitudinal study is required to address this issue. Finally, renal angiography was not performed in the patients because it was considered unethical to perform a procedure with an increased risk for acute kidney injury in a population with a low prevalence of renal artery stenosis (43). Consequently, renal artery stenosis was excluded in the patients enrolled in the present study with a non-invasive Doppler ultrasound examination (24).

In conclusion, renal vascular alterations were associated with coronary atherosclerotic burden in patients with hypertension with mild coronary disease.

\section{Acknowledgements}

None.

\section{Funding}

No funding was received.

\section{Availability of data and materials}

The datasets generated/analyzed in the current study are available on reasonable request from the corresponding author.

\section{Authors' contributions}

GG, DB and LZ have contributed substantially to the conception and design of the study, analysis and interpretation of data and drafting the paper; AG, SS, PF, SC and GM have contributed substantially to the design of the study, interpretation of data, revising the work critically for important intellectual content; KDN, MMZ, EN, CG, EM, GZ, DP and GA have contributed substantially to the acquisition of data and revising the work critically for important intellectual content. All Authors have read and approved the manuscript and agree to be accountable for all aspects of the work in ensuring that questions related to the accuracy or integrity of any part of the work are appropriately investigated and resolved.

\section{Ethics approval and consent to participate}

Written informed consent was obtained from each subject, and a local review board of the University of Palermo approved the study protocol, conformed to the ethical guidelines of Helsinki declaration.

\section{Patient consent for publication}

Not applicable.

\section{Competing interests}

The authors declare that they have no competing interests.

\section{References}

1. Huang Y, Su L, Cai X, Mai W, Wang S, Hu Y, Wu Y, Tang H and $\mathrm{Xu}$ D: Association of all-cause and cardiovascular mortality with prehypertension: A meta-analysis. Am Heart J 167: 160-168.e1, 2014.

2. Li G, Zhang P, Wang J, An Y, Gong Q, Gregg EW, Yang W, Zhang B, Shuai Y, Hong J, et al: Cardiovascular mortality, all-cause mortality, and diabetes incidence after lifestyle intervention for people with impaired glucose tolerance in the da qing diabetes prevention study: A 23-year follow-up study. Lancet Diabetes Endocrinol 2: 474-480, 2014.

3. Nakamura K, Nakagawa H, Murakami Y, Kitamura A, Kiyama M, Sakata K, Tsuji I, Miura K, Ueshima H and Okamura T; EPOCH-JAPAN research group: Smoking increases the risk of all-cause and cardiovascular mortality in patients with chronic kidney disease. Kidney Int 88: 1144-1152, 2015.

4. Nedkoff L, Knuiman M, Hung J and Briffa TG: Long-term all-cause and cardiovascular mortality following incident myocardial infarction in men and women with and without diabetes: Temporal trends from 1998 to 2009. Eur J Prev Cardiol 23: 1273-1281, 2016.

5. Xia X, He F, Wu X, Peng F, Huang F and Yu X: Relationship between serum uric acid and all-cause and cardiovascular mortality in patients treated with peritoneal dialysis. Am J Kidney Dis 64: 257-264, 2014.

6. Capodanno D, Marcantoni C, Ministeri M, Dipasqua F, Zanoli L, Rastelli S, Mangiafico S, Sanfilippo M, Romano G and Tamburino $\mathrm{C}$ : Incorporating glomerular filtration rate or creatinine clearance by the modification of diet in renal disease equation or the cockcroft-gault equations to improve the global accuracy of the age, creatinine, ejection fraction [ACEF] score in patients undergoing percutaneous coronary intervention. Int $\mathrm{J}$ Cardiol 168: 396-402, 2013.

7. Rosei EA and Muiesan ML: Early target organ damage and its reversibility: The heart. Clin Exp Hypertens 26: 673-687, 2004.

8. Tublin ME, Bude RO and Platt JF: Review. The resistive index in renal Doppler sonography: where do we stand? AJR Am J Roentgenol 180: 885-892, 2003.

9. Geraci G, Mule G, Costanza G, Mogavero M, Geraci C and Cottone S: Relationship between carotid atherosclerosis and pulse pressure with renal hemodynamics in hypertensive patients. Am J Hypertens 29: 519-527, 2016.

10. Geraci G, Mule G, Mogavero M, Geraci C, D'Ignoti D, Guglielmo C and Cottone S: Renal haemodynamics and severity of carotid atherosclerosis in hypertensive patients with and without impaired renal function. Nutr Metab Cardiovasc Dis 25: 160-166, 2015.

11. Tedesco MA, Natale F, Mocerino R, Tassinario G and Calabrò R: Renal resistive index and cardiovascular organ damage in a large population of hypertensive patients. J Hum Hypertens 21: 291-296, 2007.

12. Geraci G, Mule G, Geraci C, Mogavero M, D'Ignoto F, Morreale M, Foraci AC and Cottone S: Association of renal resistive index with aortic pulse wave velocity in hypertensive patients. Eur J Prev Cardiol 22: 415-422, 2015

13. Mule G, Geraci G, Geraci C, Morreale M and Cottone S: The renal resistive index: is it a misnomer? Intern Emerg Med 10: 889-891, 2015.

14. Doi Y, Iwashima Y, Yoshihara F, Kamide K, Takata H, Fujii T, Kubota Y, Nakamura S, Horio T and Kawano Y: Association of renal resistive index with target organ damage in essential hypertension. Am J Hypertens 25: 1292-1298, 2012.

15. Pearce JD, Craven TE, Edwards MS, Corriere MA, Crutchley TA, Fleming SH and Hansen KJ: Associations between renal duplex parameters and adverse cardiovascular events in the elderly: A prospective cohort study. Am J Kidney Dis 55: 281-290, 2010.

16. Toledo C, Thomas G, Schold JD, Arrigain S, Gornik HL, Nally JV and Navaneethan SD: Renal resistive index and mortality in chronic kidney disease. Hypertension 66: 382-388, 2015. 
17. Doi Y, Iwashima Y, Yoshihara F, Kamide K, Hayashi S, Kubota Y, Nakamura S, Horio T and Kawano Y: Renal resistive index and cardiovascular and renal outcomes in essential hypertension. Hypertension 60: 770-777, 2012.

18. Ennezat PV, Maréchaux S, Six-Carpentier M, Pinçon C, Sediri I, Delsart P, Gras M, Mounier-Véhier C, Gautier C, Montaigne D, et al: Renal resistance index and its prognostic significance in patients with heart failure with preserved ejection fraction. Nephrol Dial Transplant 26: 3908-3913, 2011.

19. Jashari F, Ibrahimi P, Nicoll R, Bajraktari G, Wester P and Henein MY: Coronary and carotid atherosclerosis: Similarities and differences. Atherosclerosis 227: 193-200, 2013.

20. Inaba Y, Chen JA and Bergmann SR: Carotid plaque, compared with carotid intima-media thickness, more accurately predicts coronary artery disease events: A meta-analysis. Atherosclerosis 220: 128-133, 2012.

21. Spence JD: Ultrasound measurement of carotid plaque as a surrogate outcome for coronary artery disease. Am J Cardiol 89: 10B-15B; discussion 15B-16B, 2002.

22. Sinha AK, Eigenbrodt M and Mehta JL: Does carotid intima media thickness indicate coronary atherosclerosis? Curr Opin Cardiol 17: 526-530, 2002.

23. Komorovsky R and Desideri A: Carotid ultrasound assessment of patients with coronary artery disease: A useful index for risk stratification. Vasc Health Risk Manag 1: 131-136, 2005.

24. Granata A, Fiorini F, Andrulli S, Logias F, Gallieni M, Romano G, Sicurezza E and Fiore CE: Doppler ultrasound and renal artery stenosis: An overview. J Ultrasound 12: 133-143, 2009.

25. Mancia G, Fagard R, Narkiewicz K, Redon J, Zanchetti A, Böhm M, Christiaens T, Cifkova R, De Backer G, Dominiczak A, et al: 2013 ESH/ESC guidelines for the management of arterial hypertension: the task force for the management of arterial hypertension of the european society of hypertension (ESH) and of the european society of cardiology (ESC). Eur Heart J 34: 2159-2219, 2013.

26. Lang RM, Badano LP, Mor-Avi V, Afilalo J, Armstrong A, Ernande L, Flachskampf FA, Foster E, Goldstein SA, Kuznetsova T, et al: Recommendations for cardiac chamber quantification by echocardiography in adults: An update from the american society of echocardiography and the european association of cardiovascular imaging. Eur Hear J Cardiovasc Imaging 16: 233-270, 2015.

27. Gensini GG: A more meaningful scoring system for determining the severity of coronary heart disease. Am J Cardiol 51: 606, 1983.

28. Stergiou GS, Tzamouranis D, Protogerou A, Nasothimiou E and Kapralos C: Validation of the microlife watch BP office professional device for office blood pressure measurement according to the International protocol. Blood Press Monit 13: 299-303, 2008

29. Levey AS, Stevens LA, Schmid CH, Zhang YL, Castro AF III, Feldman HI, Kusek JW, Eggers P, Van Lente F, Greene T, et al: A new equation to estimate glomerular filtration rate. Ann Intern Med 150: 604-612, 2009

30. Nazzal MM, Hoballah JJ, Miller EV, Sharp WJ, Kresowik TF and Corson J: Renal hilar doppler analysis is of value in the management of patients with renovascular disease. Am J Surg 174: 164-168, 1997

31. Granata A, Zanoli L, Clementi S, Fatuzzo P, Di Nicolò P and Fiorini F: Resistive intrarenal index: Myth or reality? Br J Radiol 87: 20140004, 2014

32. Touboul PJ, Hennerici MG, Meairs S, Adams H, Amarenco P, Bornstein N, Csiba L, Desvarieux M, Ebrahim S, Hernandez Hernandez R, et al: Mannheim carotid intima-media thickness and plaque consensus (2004-2006-2011). An update on behalf of the advisory board of the 3rd, 4th and 5th watching the risk symposia, at the 13th, 15th and 20th European stroke conferences, Mannheim, Germany, 2004, Brussels, Belgium, 2006, and Hamburg, Germany, 2011. Cerebrovasc Dis 34: 290-296, 2012.
33. Neale ML, Chambers JL, Kelly AT, Connard S, Lawton MA, Roche J and Appleberg M: Reappraisal of duplex criteria to assess significant carotid stenosis with special reference to reports from the North American symptomatic carotid endarterectomy trial and the European carotid surgery trial. J Vasc Surg 20: 642-649, 1994.

34. Polak JF, Szklo M, Kronmal RA, Burke GL, Shea S, Zavodni AE and O'Leary DH: The value of carotid artery plaque and intima-media thickness for incident cardiovascular disease: The multi-ethnic study of atherosclerosis. J Am Heart Assoc 2: e000087, 2013.

35. Wilson PW, Hoeg JM, D'Agostino RB, Silbershatz H, Belanger AM, Poehlmann H, O'Leary D and Wolf PA: Cumulative effects of high cholesterol levels, high blood pressure, and cigarette smoking on carotid stenosis. N Engl J Med 337: 516-522, 1997.

36. Smith SC Jr, Dove JT, Jacobs AK, Kennedy JW, Kereiakes D, Kern MJ, Kuntz RE, Popma JJ, Schaff HV, Williams DO, et al: ACC/AHA guidelines for percutaneous coronary intervention (revision of the 1993 PTCA guidelines)-executive summary: A report of the American college of cardiology/American Heart Association task force on practice guidelines (committee to revise the 1993 guidelines for percutaneous transluminal coronary angioplasty) endorsed by the society for cardiac angiography and interventions. Circulation 103: 3019-3041, 2001.

37. Krämer B, Brill M, Brühn A and Kübler W: Relationship between the degree of coronary artery disease and of left ventricular function and the duration of the QT-interval in ECG. Eur Heart J 7: 14-24, 1986.

38. Zanoli L, Rastelli S, Marcantoni C, Tamburino C, Laurent S, Boutouyrie P and Castellino P: Renal artery diameter, renal function and resistant hypertension in patients with low-to-moderate renal artery stenosis. J Hypertens 30: 600-607, 2012.

39. Zanoli L, Rastelli S, Marcantoni C, Capodanno D, Blanco J, Tamburino C, Laurent S, Boutouyrie P and Castellino P: Non-hemodynamically significant renal artery stenosis predicts cardiovascular events in persons with ischemic heart disease. Am J Nephrol 40: 468-477, 2014.

40. Bigé N, Lévy PP, Callard P, Faintuch JM, Chigot V, Jousselin V, Ronco P and Boffa JJ: Renal arterial resistive index is associated with severe histological changes and poor renal outcome during chronic kidney disease. BMC Nephrol 13: 139, 2012.

41. Florczak E, Januszewicz M, Januszewicz A, Prejbisz A, Kaczmarska M, Michałowska I, Kabat M, Rywik T, Rynkun D, Zieliński T, et al: Relationship between renal resistive index and early target organ damage in patients with never-treated essential hypertension. Blood Press 18: 55-61, 2009.

42. Granata A, Clementi A, Virzì GM, Brocca A, de Cal M, Scarfia VR, Zanoli L, Ronco C, Corrao S and Malatino L: Cardiorenal syndrome type 4: From chronic kidney disease to cardiovascular impairment. Eur J Intern Med 30: 1-6, 2016.

43. Marcantoni C, Rastelli S, Zanoli L, Tripepi G, Di Salvo M, Monaco S, Sgroi C, Capodanno D, Tamburino C and Castellino P: Prevalence of renal artery stenosis in patients undergoing cardiac catheterization. Intern Emerg Med 8: 401-408, 2013. 